\title{
Evidence for Prenatal Competition among the Central Arbors of Trigeminal Primary Afferent Neurons
}

\author{
Nicolas L. Chiaia, Carol A. Bennett-Clarke, Marcia Eck, Fletcher A. White, Robert S. Crissman, and \\ Robert W. Rhoades \\ Department of Anatomy, Medical College of Ohio, Toledo, Ohio 43699
}

Previous studies have shown that damage to vibrissa follicles in newborn rats and mice does not alter the brainstem representations of the remaining vibrissa as demonstrated by staining for mitochondrial enzymes such as cytochrome oxidase (CO) succinic dehydrogenase. This study asked whether this lack of effect might be due to the fact that the trigeminal primary afferents in rodents are already quite well developed at birth. We assessed this possibility by using CO staining to evaluate patterns in the brainstems of preand postnatal rats. A vibrissa-related pattern began to emerge in trigeminal nucleus principalis and subnucleus interpolaris (Spl) by embryonic day (E-) 19 and appeared fully developed by the day of birth (P-0). We also made partial lesions of the vibrissa pad on E-15-20 and on P-0, killed pups on P-5-7, and measured the size of the CO-stained patches in Spl on both sides of the brainstem. The correspondence between $\mathrm{CO}$ patches and clusters of primary afferent terminal arbors was verified in some animals by combining transganglionic horseradish peroxidase tracing and $\mathrm{CO}$ staining. Vibrissa pad damage on E-15-18 resulted in significant (20.1-36.9\%) increases in the average area of the remaining $C O$ patches in Spl ipsilateral to the lesion. Vibrissa pad damage on E-19, $E-20$, and P-O produced small (6.2-8.9\%), but insignificant, increases in patch size in Spl ipsilateral to the lesion. We used anatomical and electrophysiological methods to determine whether our lesions altered the trigeminal innervation of surviving vibrissa follicles. We recorded single trigeminal ganglion cells from 12 rats that sustained vibrissa pad lesions on E-17. As in normal rats, all of the 49 vibrissasensitive ganglion cells isolated in the lesioned animals were responsive to deflection of one and only one vibrissa. We also dissected 11 deep vibrissal nerves from intact follicles in adult rats that sustained fetal vibrissa pad damage on $\mathrm{E}-17$, and counted numbers of myelinated axons in $1 \mu \mathrm{m}$ plastic sections. These data were compared with counts from corresponding follicles on the intact side of the face. The average number of myelinated axons innervating follicles in the damaged vibrissa pads was $196.8 \pm 27.9$, and that for the corresponding contralateral nerves was $194.6 \pm$ 25.7. These data suggest that competitive interactions among the central arbors of trigeminal primary afferents in fetal life may influence the development of central vibrissa repre-

Received May 23, 1991; revised July 23, 1991 ; accepted July 30, 1991.

This work was supported by NS 2888 DE 07734 , and DE 08971 . Thanks to Beth Figley, Shelly Haube, and Ann Marie Eckles for excellent technical assistance. Correspondence should be addressed to Dr. Nicolas L. Chiaia, Department of Anatomy, Medical College of Ohio, CS\# 10008, Toledo, OH 43699.

Copyright (C) 1992 Society for Neuroscience $0270-6474 / 92 / 120062-15 \$ 05.00 / 0$ sentations and, further, that lesion-induced central changes need not be correlated with alterations in the peripheral innervation of undamaged follicles.

Development of the mature size and location of the terminal arbors of axons innervating a given target is thought to involve interactions among these fibers in which they compete for some limited resource provided by that target. Such interactions are imputed when ablation or reduction in the activity of one set of afferents results in increased terminal arborizations of fibers that remain or retain normal patterns of activity (Sherman and Spear, 1982). Interaxonal competition has been indicated to occur in the development of the neuromuscular junction (Betz et al., 1980; Bennett et al., 1986), the primary afferent innervation of the skin (e.g., Scott, 1984; Smith and Frank, 1988), and both retinofugal (e.g., Lund and Lund, 1971; Rakic, 1981; Chalupa and Williams, 1984; Sretavan and Shatz, 1986; Coleman and Beazley, 1989) and geniculocortical (Wiesel and Hubel, 1963; Guillery, 1972a; LeVay et al., 1980) projections.

Existing data suggest that such interactions may not play a role in the trigeminal primary afferent innervation of the brainstem. A number of investigators have used cautery of subsets of vibrissa follicles to assay the effects of peripheral lesions upon trigeminal primary afferent development. Both Belford and Killackey (1980) and Durham and Woolsey (1984) employed this paradigm in conjunction with staining for succinic dehydrogenase $(\mathrm{SDH})$ or cytochrome oxidase $(\mathrm{CO})$ to demonstrate the patches corresponding (Bates and Killackey, 1985) to the trigeminal primary afferent innervation of the brainstem. Both of these studies produced the same result: there was no expansion of the terminal arbors of undamaged afferents into territory normally occupied by axons projecting to the damaged follicles. These results led both Belford and Killackey (1980) and Durham and Woolsey (1984) to conclude that competition was not likely to play a role in shaping the postnatal development of the primary afferent innervation of the trigeminal brainstem complex.

One important limitation of these experiments is that surgical manipulation of the afferent input to the brainstem was carried out only after birth. Both CO staining (Erzurumlu and Killackey, 1983) and transganglionic transport of horseradish peroxidase (HRP) (Jacquin and Rhoades, 1985) have shown that the trigeminal innervation of the brainstem has a rclativcly adultlike organization in newborn rats. It may thus be that the lesions carried out by Belford and Killackey (1980) and Durham and Woolsey (1984) were simply too late to demonstrate competitive interactions among trigeminal primary afferents innervating the whisker pad. Data from a number of systems have demonstrated that there are limited sensitive or critical periods after which 
experimental manipulations have relatively limited effects upon axonal development (compare the results of Chalupa and Williams, 1984, and Sretaven and Shat7, 1986, with those of Guillery, $1972 \mathrm{~b}$, with respect to the effects of pre- and postnatal enucleation upon the innervation of the LGNd by axons from the remaining eye).

In an effort to determine whether competitive interactions during prenatal development play a role in shaping the primary afferent innervation of the trigeminal brainstem complex, we made peripheral lesions in rats on embryonic days (E-) 15-20 and on the day of birth (P-O) and used $\mathrm{CO}$ staining and transganglionic HRP transport to determine whether or not such lesions altered the remaining trigeminal innervation of the brainstem near the end of the first postnatal week. In order to determine whether changes observed in brainstem were associated with alterations in the peripheral innervation patterns of surviving trigeminal ganglion cells, a small number of adult rats that sustained prenatal peripheral damage were used to assess the numbers of myelinated axons in follicle nerves and the receptive fields of individual trigeminal (V) ganglion cells.

\section{Materials and Methods}

Experimental animals. Ninety-eight perinatal and 15 adult SpragueDawley rats from 22 litters provided data for this study. Timed pregnancies were obtained by placing two females with an experienced male at the start of the animal colony's dark cycle (7:00 P.M.). The animals were separated the following morning, and vaginal smears were examined for the presence of sperm. In the case of a sperm-positive smear, conception was considered to have occurred the previous evening and this day was designated E- 0 .

Fetal follicle cauterization. Mystacial vibrissa follicles were cauterized in different litters on E-15-20 and on the day of birth (P-0). Fetal whisker follicle cauterizations were accomplished by anesthetizing pregnant females with ether, making a midline abdominal incision, and exteriorizing the uterine horns. The uterine horns were transilluminated with a fiber-optic light and viewed through a dissecting microscope. A parylene-coated tungsten microelectrode attached to the wand of an electrocautery device (Birtcher, model 732 Hyfrecator, $750 \mathrm{kHz}$ spark gap oscillator) was inserted through the uterine wall and amniotic sac to contact the left vibrissa pad. The electrocautery was then activated for 2 sec. Following follicle cauterization of all embryos in a litter, the uterine horns were replaced and the abdominal incision in the dam was sutured closed. Manipulated pups were allowed to come to term. Animals used in the recording experiments and for counts of follicle nerves survived at least $45 \mathrm{~d}$. All other animals were killed on P-5-7.

Cytochrome oxidase staining. All perinatal animals were anesthetized with ether and perfused transcardially with sodium phosphate-buffered saline (pH 7.4) followed by $4 \%$ paraformaldehyde in the same buffer. Brains were removed, and frozen $50 \mu \mathrm{m}$ coronal sections of the trigeminal brainstem complex were cut and collected in phosphate-buffered saline (PBS; pH 7.4). Free-floating sections were transferred into freshly prepared $C O$ incubation medium that contained $75 \mathrm{mg}$ of diaminobenzidine, $35 \mathrm{mg}$ of cytochrome C (type III, Sigma), and $6 \mathrm{gm}$ of sucrose in $130 \mathrm{ml}$ of $0.1 \mathrm{M}$ phosphate buffer (PB; pH 7.4) (Wong-Riley, 1979). The incubation solution was saturated for $5 \mathrm{~min}$ with $5 \% \mathrm{CO}_{2} / 95 \% \mathrm{O}_{2}$. The incubation was carried out at $37^{\circ} \mathrm{C}$ for $12-24 \mathrm{hr}$ and arrested with several rinses in PBS when there was clear differentiation of "patches" in the brainstem. Stained sections were rinsed in PBS and plated out of gel-alcohol solution onto glass slides. Slides were allowed to air dry, dehydrated in $100 \%$ alcohol, cleared in xylene, and coverslipped with Permount.

In order to evaluate the normal development of the vibrissa-related $\mathrm{CO}$ pattern in the trigeminal brainstem complex, fetuses (at least four from each age) from rats E-15 through $P-1$ were harvested, anesthetized with ether, and perfused in the manner described above. The brainstems of these animals were processed for $\mathrm{CO}$ as described above.

Staining of damaged vibrissa pads. In order to assess the extent of the damage to the periphery, the facial skin from the lesioned animals was removed, dehydrated in graded alcohols, and embedded in paraffin. Fifteen micrometer sections were cut through the vibrissa pad parallel to the skin surface. Alternate series of sections were stained with standard hematoxylin and eosin or a reduced silver stain for nerve fibers (Ungewitter, 1951).

Data analysis. Cytochrome oxidase-stained sections were examined with a Nikon Optiphot microscope equipped with a drawing tube. Sixtysix brains in which the vibrissae representations in the trigeminal brainstem complex could be completely and unambiguously reconstructed from serial sections were chosen for analysis. Areal analysis of the vibrissae-related cytochrome oxidase pattern was carried out in subnucleus interpolaris (SpI). Of the three representations of the vibrissae in the trigeminal brainstem complex, the pattern in this subnucleus is the easiest to discern due to its large volume and the high contrast between the patches and "interpatch" regions (Belford and Killackey, 1980).

Patch perimeters on both sides of the brainstem were drawn from six to eight serial sections through SpI. On the intact side, only the patches corresponding to the four most caudal vibrissae $(1-4)$ in rows A-E were drawn. On the lesioned side, as many of these same patches as could be unambiguously identified also were drawn. Measurements of the cross-sectional areas of patches in these reconstructions were obtained with the aid of a graphics tablet. The mean cross-sectional area of $\mathrm{CO}$ patches (total area measured/number of patches measured) for each side was calculated. The percent difference between the average cross-sectional areas on the two sides was then determined.

The resultant values were then used in a one-way analysis of covariance, with age at the time of lesion as the independent variable and the number of patches on the side of the brainstem ipsilateral to the damaged whisker pad as the covariate. Post hoc evaluations of the percent changes in rats lesioned at a given embryonic age were made by means of Scheffe tests.

Because previous results from thalamus and cortex (Woolsey and Wann, 1976; Durham and Woolsey, 1984) indicated that patches in rows adjacent to the one that was damaged were more likely to increase in size than those in more distant rows, we carried out one additional analysis. Here, cases in which damage was restricted to one or two rows of vibrissae rather than a portion of the follicles in most or all rows were analyzed on a row-by-row basis. The results were then used to carry out a one-way analysis of variance, with the number of rows between the damaged row and the row analyzed as the independent variable. Post hoc comparisons here were also made by means of Scheffe tests. The level of statistical significance for all tests was set at $p<0.05$.

To determine whether the changes observed in SpI were also apparent in the principal trigeminal sensory nucleus (PrV), measurements of $\mathrm{CO}$ stained patches were carried out in 13 brains (from the above sample) in which the pattern in this nucleus was clear.

Horseradish peroxidase labeling of $V$ primary afferents. In order to determine the relationship between patches of $\mathrm{CO}$ reactivity and trigeminal primary afferents in rats that sustained fetal follicle cauterizations, these axons were directly labeled in five rats aged P-5 that sustained follicle damage on E-16. Six injections $(1 \mu \mathrm{l}$ each) of an HRP cocktail containing $6 \%$ wheat germ agglutinin-conjugated HRP (Sigma), $0.5 \%$ cholera toxin HRP (List Biological Lab), and $10 \%$ free HRP (Sigma type VI) were made throughout the lesioned whisker pad using a pipette with a $30-50 \mu \mathrm{m}$ tip. Following a $48 \mathrm{hr}$ survival time, animals were anesthetized with ether and perfused transcardially with physiological saline, a fixative solution containing $2 \%$ paraformaldehyde, and $1.5 \%$ glutaraldehyde in sodium phosphate buffer, and a $10 \%$ buffered sucrose rinse. Brains were removed and stored overnight at $4^{\circ} \mathrm{C}$ in $10 \%$ buffered sucrose. Coronal sections through the brainstem were cut at $50 \mu \mathrm{m}$ on a freezing microtome. Alternate sections were processed for HRP reaction product according to the method of Mesulam (1978) or for $\mathrm{CO}$ reactivity using the methods described above.

Recording from V ganglion cells. Twelve adult rats that sustained fetal follicle cauterizations were anesthetized with sodium pentobarbital ( 60 $\mathrm{mg} / \mathrm{kg}$, i.p., with $0.1 \mathrm{ml}$ of $1.5 \%$ atropine sulfate), the trachea was cannulated, heart rate recording leads were fastened to the chest, and the rat was then placed in a stereotaxic apparatus. Following a midline incision in the overlying skin, the dorsal calvarium was removed from the side ipsilateral to the damaged whisker pad, the forebrain aspirated and the trigeminal ganglion exposed. Wound edges were infiltrated with a long-lasting local anesthetic (Nupercaine, CIBA). The rat was then paralyzed with $10 \mathrm{mg}$ of gallamine triethiodide, and artificial respiration was initiated. Anesthesia and paralysis were maintained with hourly doses of sodium pentobarbital and gallamine triethiodide $(10 \mathrm{mg}$ and $4 \mathrm{mg}$, respectively). Body temperature was held at $37 \pm 1^{\circ} \mathrm{C}$ with a feedback-controlled heating blanket. 

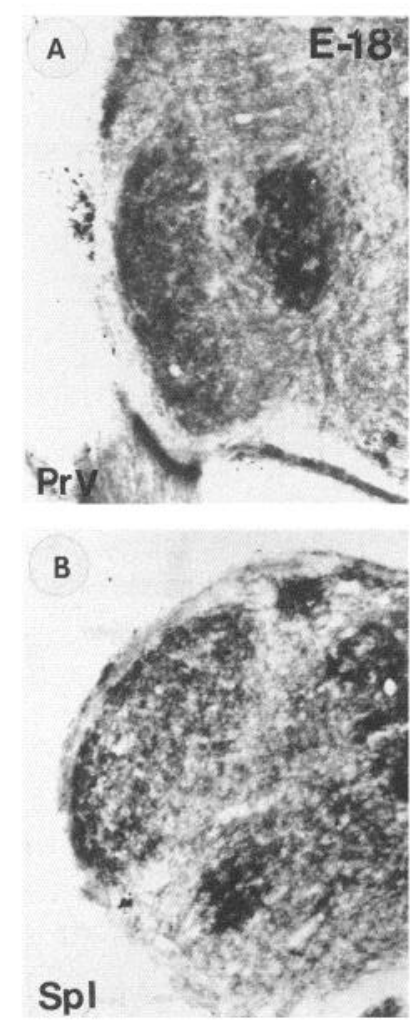
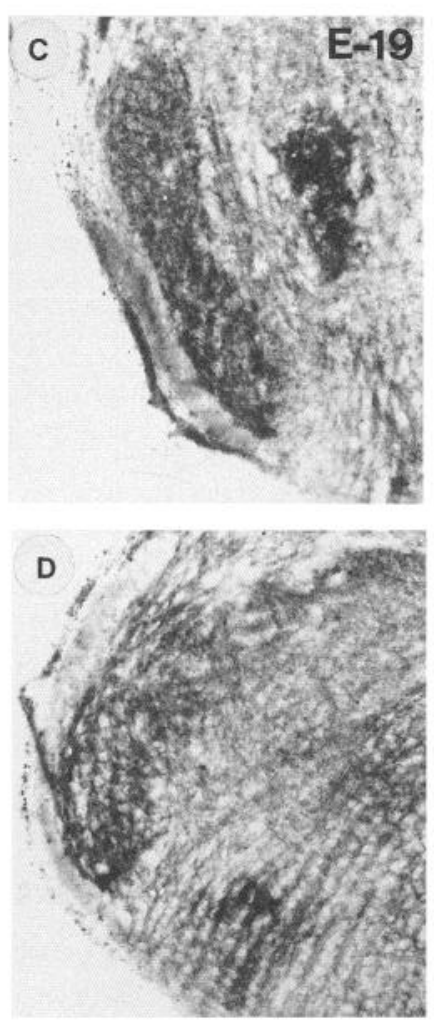
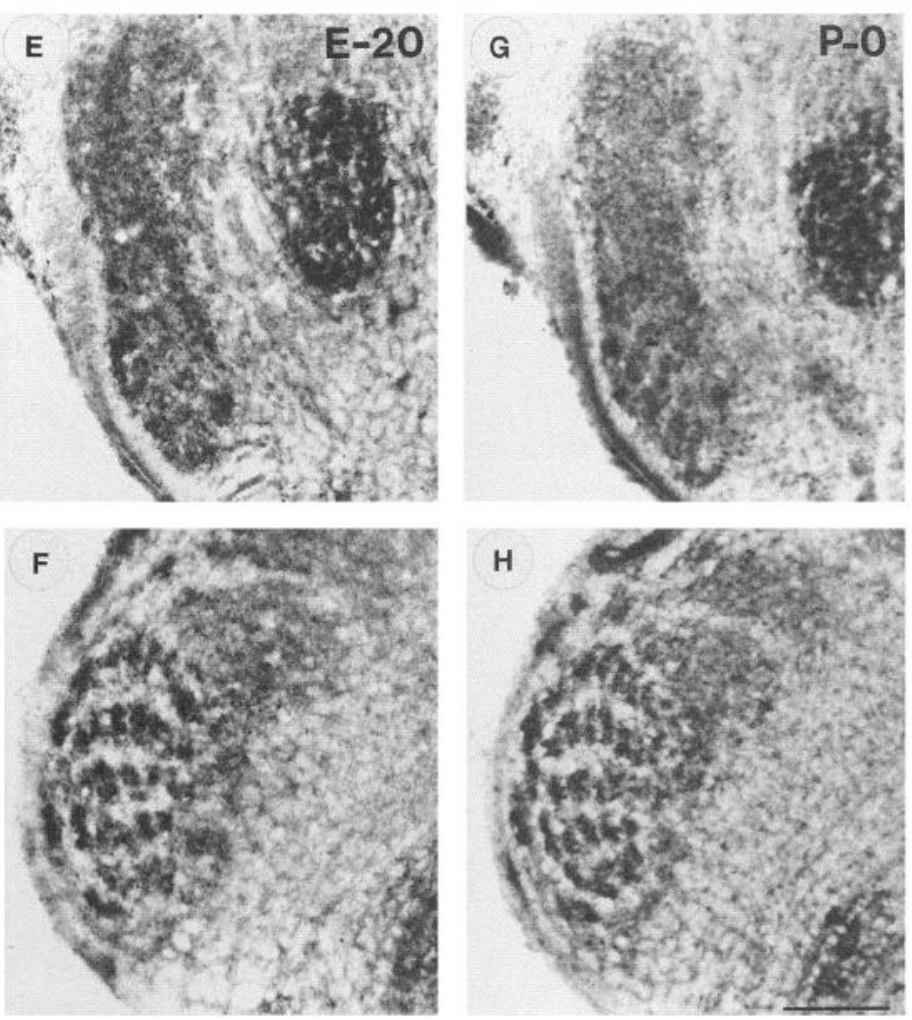

Figure 1. CO-stained sections through PrV and SpI of rats killed on E-18 through P-0. On E-18 ( $A$ and $B$ ), there is dense CO staining in PrV and SpI, but no pattern is apparent. On E-19 $(C$ and $D)$, segmentation is apparent within the dense staining in PrV and SpI, but the pattern cannot be related to the pattern of the vibrissae. On E-20 $(E$ and $F)$, the pattern of CO staining is somewhat more distinct, and by P-0 $(G$ and $H)$, the pattern observed in older perinatal rats is clearly present. Scale bar, $100 \mu \mathrm{m}$ for all panels.

The effectiveness of this anesthetic regimen was evaluated in two ways. First, it was used in rats that were not paralyzed. Such animals lie quietly and are behaviorally unresponsive to external stimuli for at least $1 \mathrm{hr}$ after discontinuance of sodium pentobarbital. Second, rats were allowed to recover from paralysis during the recording experiments, and it was determined that anesthesia was still sufficient to prevent responses to mechanical stimulation.

Tungsten electrodes (impedance, 20-25 M 2 ) were visually positioned over the ophthalmic/maxillary portion of the ganglion. The electrodes were slowly advanced through the ganglion with a hydraulic microdrive, and extracellular recordings were amplified and displayed using standard methods. Stimulation was delivered to the whisker pad with hand-held probes using previously described methods (Jacquin et al., 1986). The response properties of each well-isolated unit were determined, and the receptive field was carefully mapped. Particular attention was paid to vibrissa-sensitive units. For these, we determined both the total number of whiskers to which the unit responded and whether or not skin or surrounding guard hairs were included in the receptive field.

The extent of the follicle damage in each of these rats was determined by histological processing of the vibrissa pad using the methods described above and CO staining of the cortex contralateral to the damaged side of the face. The vibrissa-related CO pattern is difficult to see in the brainstems of adult rats, but the pattern remains quite apparent in $\mathrm{CO}$ stained sections through cortical lamina IV.

Counts of myelinated axons in nerves supplying spared follicles. Three adult rats that sustained vibrissa pad lesions on E-17 were anesthetized with sodium pentobarbital $(60 \mathrm{mg} / \mathrm{kg}$, i.p. $)$ and perfused transcardially with a washout solution $(0.1 \mathrm{~m}$ cacodylate buffer containing $0.4 \% \mathrm{xy}-$ locaine and $0.4 \%$ heparin, $\mathrm{pH} 7.4$ ) followed by a buffered fixative solution (3\% glutaraldehyde and 3\% paraformaldehyde in $0.1 \mathrm{M}$ cacodylate buffer, $\mathrm{pH}$ 7.4). Brains were removed, and $50-\mu \mathrm{m}$-thick frozen sections of flattened cortex were cut and reacted for the demonstration of $\mathrm{CO}$ in the manner described above. Spared individual vibrissa follicles corresponding to enlarged $\mathrm{CO}$ cortical patches as well as the contralateral normal follicles were dissected from the mystacial pad, rinsed in cacodylate buffer, postfixed for $2 \mathrm{hr}$ in $1.5 \%$ osmium tetroxide and $1.5 \%$ potassium ferricyanide in $0.1 \mathrm{M}$ cacodylate buffer $(\mathrm{pH} 7.4)$, dehydrated in a graded series of ethanols, cleared in propylene oxide, and embedded in an Epon-Araldite mixture. Blocks were oriented so that the deep vibrissal nerve was cut perpendicular to its longitudinal axis at the level where the nerve entered the follicle capsule. One-micron-thick sections were cut and stained with toluidine blue. Myelinated axons were counted with the light microscope at $400 \times$. Each nerve bundle was counted three times, and the modal result was taken as the final fiber count.

\section{Results}

\section{Normal development of the vibrissa-related CO pattern in the trigeminal brainstem complex}

The earliest age at which any pattern could be discerned in the CO staining of the sections through either PrV or SpI was E-19 (Fig. $1 C, D$ ). While the pattern at this age could not be related to the vibrissa on a one-for-one basis, there was segmentation

Figure 2. $A$ and $B$ show the damaged and normal vibrissa pads (hematoxylin and eosin stain) of a rat that sustained a lesion on E-15 and was killed on P-7. On the lesioned side, only rows $D$ and $E$ remain. All of these follicles were innervated. $C$ and $D$, and $E$ and $F$ show CO-stained sections through PrV and SpI, respectively. The insets are low-power photographs showing both sides of the same brainstem section. Note that only two distinct rows of dense patches of $\mathrm{CO}$ reactivity remain in $\mathrm{PrV}$ and $\mathrm{SpI}$ ipsilateral to the lesion. The arrows on the sections through the vibrissa pads $(A$ and $B$ ) point to dorsal $(D)$ and anterior $(A)$. Scale bar, $200 \mu \mathrm{m}$ for all panels. 

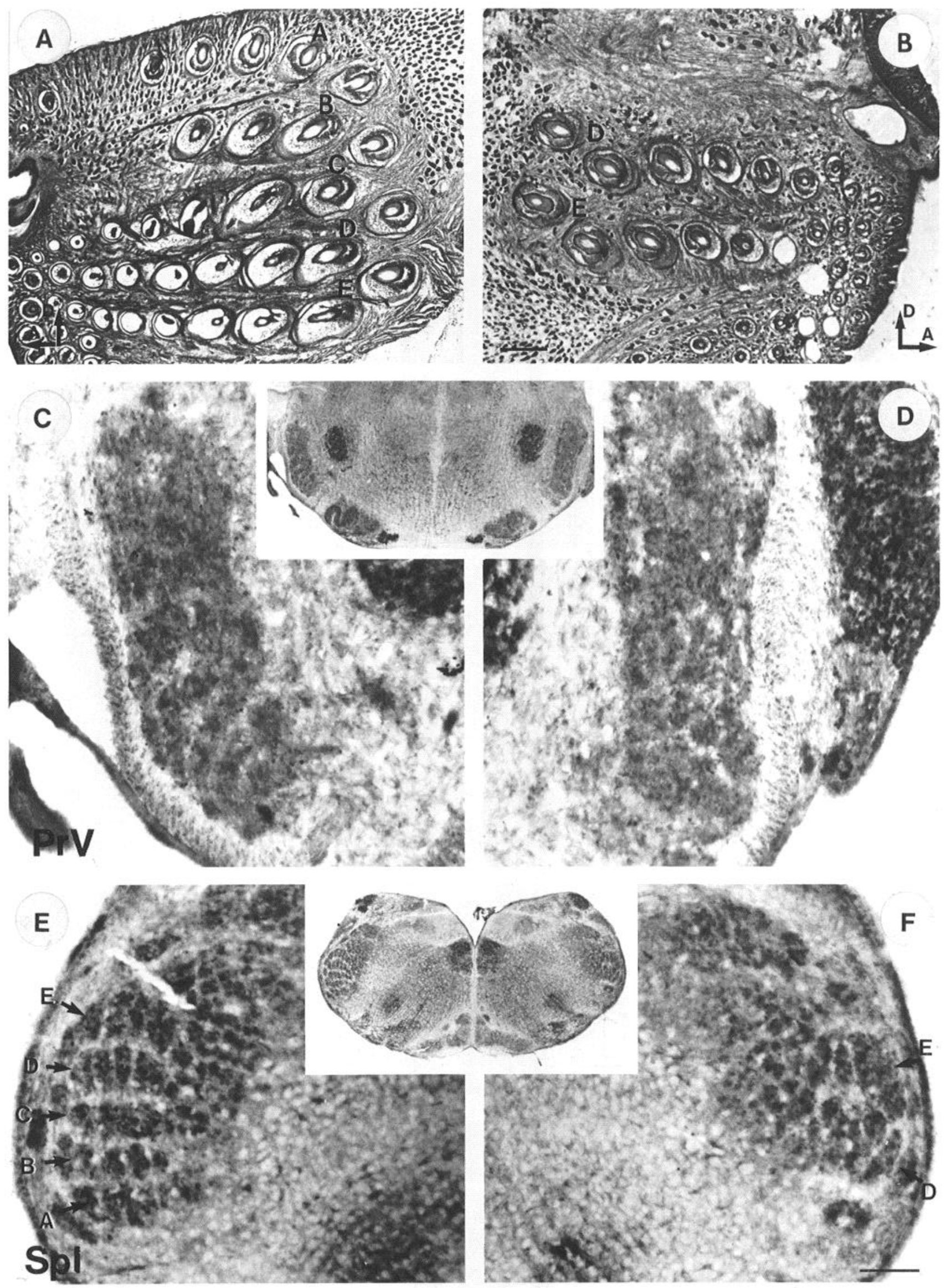

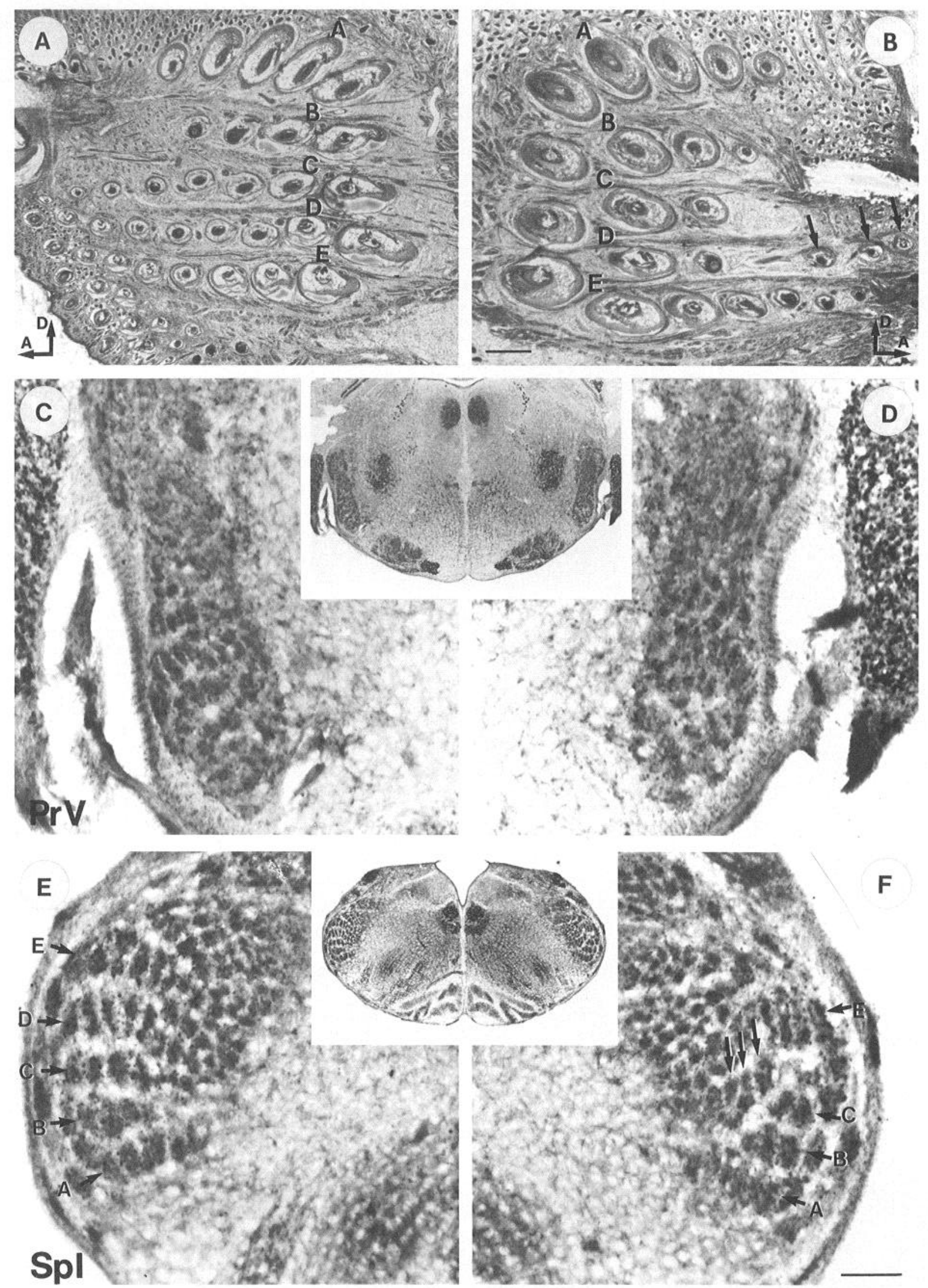

Figure 3. $A$ and $B$ show the damaged and normal vibrissa pads (hematoxylin and eosin stain) of a rat that sustained a lesion on E-16 and was killed on P-5. On the lesioned side, rows $A, B$, and $E$ are intact, as are the two most caudal follicles in row $C$ and the D1 follicle. The small rostral follicles in row $D$ (arrows) were also innervated. $C$ and $D$, and $E$ and $F$ show CO-stained sections through $\operatorname{PrV}$ and $\operatorname{SpI}$, respectively. The insets are low-power photographs showing both sides of the same brainstem section. Note the correspondence between the pattern of innervated follicles on the face and the patches of $\mathrm{CO}$ reactivity in the ipsilateral brainstem. The arrows on the sections through the vibrissa pads $(A$ and $B)$ point to dorsal $(D)$ and anterior $(A)$. Scale bar, $200 \mu \mathrm{m}$ for all panels. 

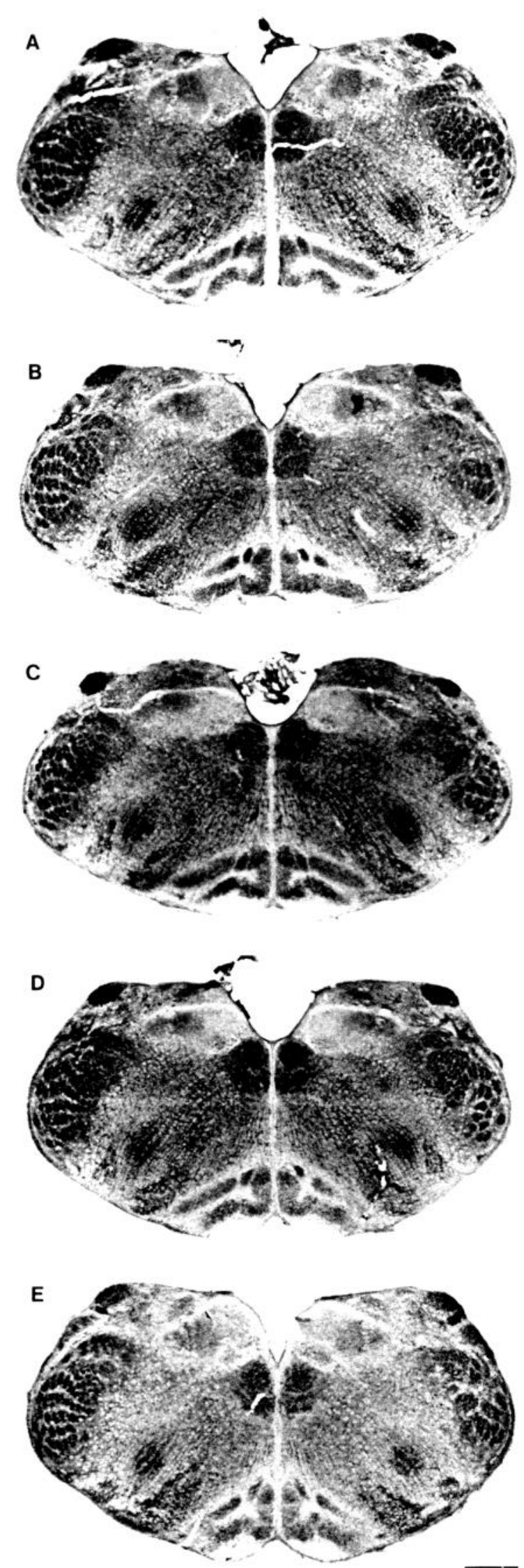

in both PrV and SpI. The segmentation within both of these nuclei became more distinct on E-20 (Fig. 1E,F) and appeared fully developed by P-0 (Fig. $1 G, H$ ).

\section{Effects of fetal vibrissa follicle damage upon CO staining patterns in the $V$ brainstem complex}

Electrocautery of a subset of vibrissa follicles on E-15-18 resulted in significant increases in the average size of the remaining patches of high $\mathrm{CO}$ density in the brainstem ipsilateral to the damaged vibrissa pad. Such increases were observed in both PrV and SpI, but for the technical reasons discussed above (Materials and Methods) only SpI was evaluated in most cases. The age at which rats were killed (P-5, P-6, or P-7) had no significant effect upon results, so all data from animals that sustained vibrissa pad damage on a given embryonic day were pooled for analysis.

Figure 2 shows results typical of those obtained from rats that sustained follicle cautery between E-15 and E-18. In this case, the lesion produced a loss of all the follicles in rows A-C (Fig. $2 B$ ) and there was an absence of the corresponding patches of high CO reactivity in both PrV (Fig. $2 D$ ) and SpI (Fig. $2 F$ ). Measurements in PrV and SpI showed increases of $35 \%$ and $44 \%$, respectively, in the average cross-sectional area of the patches that remained on the lesioned side, relative to the large vibrissa-related patches on the intact side.

In many cases (Fig. 3), the damage to the vibrissa pad was less extensive than that illustrated in Figure 2. In these rats, many more follicles remained in the damaged vibrissa pad (Fig. $3 B$ ) and the reorganization in the brainstem was much less dramatic (Fig. $3 C-F$ ). For the case shown, the increases in average patch size in PrV and SpI ipsilateral to the lesion were $21 \%$ and $18 \%$, respectively.

Further examples of the lesion-induced alterations in the $\mathrm{CO}$ staining pattern in SpI are shown in Figure 4, and the results from all $(N=66)$ of the cases included in the report are summarized in Figures 5 and 6 and in Table 1. The data presented support several conclusions. First (Fig. 5A), cautery of vibrissa follicles on E-15-18, but not at later ages, resulted in significant increases in the average cross-sectional area of the patches of high $\mathrm{CO}$ activity that remained in SpI ipsilateral to the damaged vibrissa pad (analysis of covariance: $F=14.8 ; \mathrm{df}=6,58 ; p<$ 0.0001 ; post hoc Sheffe tests all $p<0.05$ ). Second, for the E-1518 animals, there was a significant negative correlation $(r=$ $-0.65 ; p<0.01$ ) between the number of patches that remained in SpI ipsilateral to the lesion and the increase in average patch size. Scatter plots illustrating the correlations for each of these ages are presented in Figure 6. The correlation for the animals that sustained lesions on E-15 (Fig. $6 A$ ) was -0.82 but was not statistically significant because of the small number of cases in this group. The correlation for the rats lesioned on E-16 (Fig. $6 B)$ was $-0.76(p<0.05)$, that for the rats that sustained lesions on E-17 (Fig. $6 C$ ) was $-0.70(p<0.05)$, and that for the rats lesioned on E-18 (Fig. $6 D$ ) was -0.43 (not significant). Importantly, there was no significant correlation between the age at which lesions were sustained and the number of patches that remained in PrV and SpI ipsilateral to the damaged vibrissa

Figure 4. CO-stained sections through $\mathrm{SpI}$ from five different rats $(A-$ $E$ ) that sustained damage to the vibrissa pad during fetal life. Note the reduced number and increased size of the patches on the deafferented side of the brainstem. Scale bar, $500 \mu \mathrm{m}$ for all panels. 

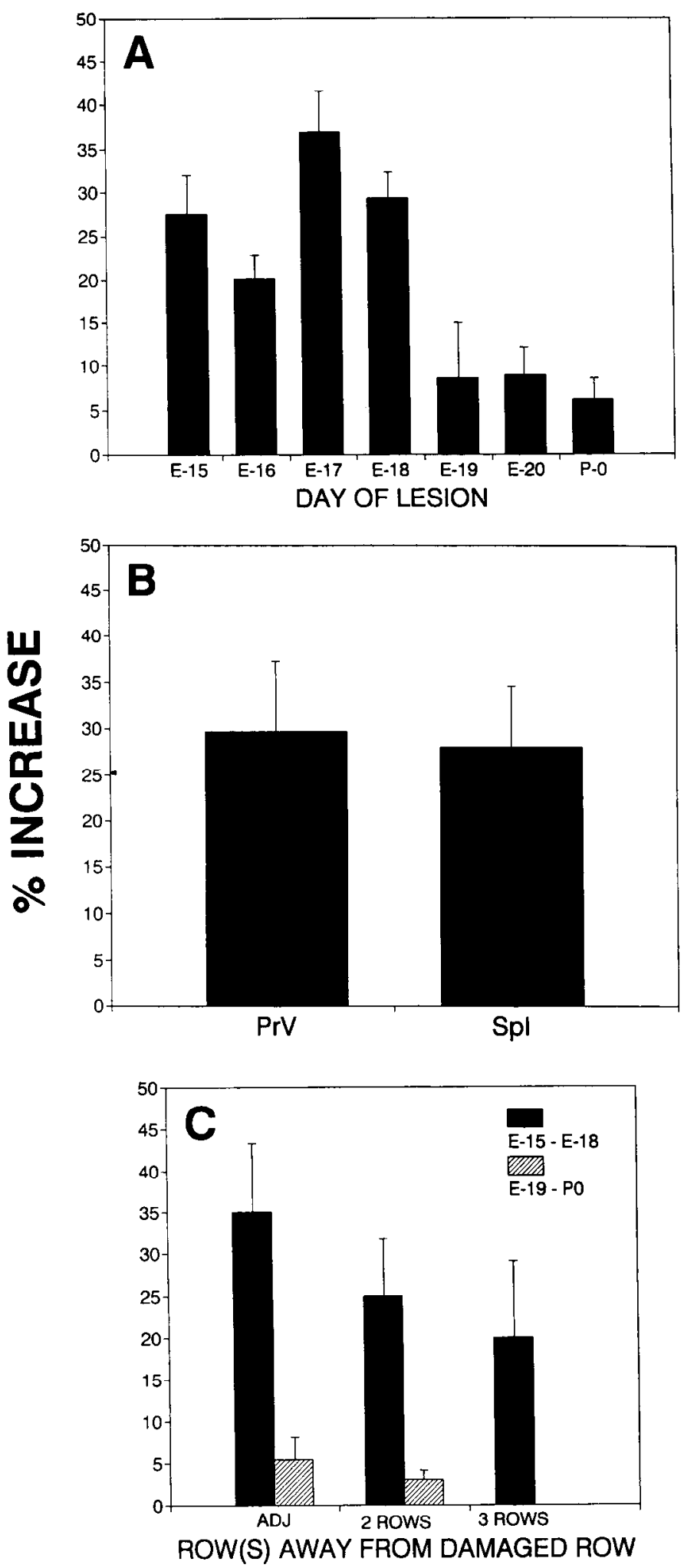

Figure 5. A shows the increase (mean \pm SEM) in average CO patch size in Spl of rats killed on P-6 or P-7 that sustained vibrissa pad damage on either E-15-20 or P-0. The increases in the animals that sustained lesions on E-15-18 were statistically significant, and those for the rats lesioned on E-19, E-20, and P-0 were not (analysis of covariance; $F=$ $14.8 ; \mathrm{df}=6,58 ; p<0.00001$; post hoc Sheffe tests, all $p<0.05$ ). The numbers of animals in each group are listed in Table $1 . B$ shows the average increases (mean \pm SEM) in CO patch size for 14 cases in which measurements were made in both PrV and SpI. There was no significant difference between the average increases in these two nuclei. $C$ shows pad. The increases in average patch size and average number of patches remaining ipsilateral to the lesion for each age group are summarized in Table 1. Finally, for the smaller number ( $N$ $=14$ ) of animals in which measurements were made in both PrV and SpI, the average increases in the two nuclei were in very close agreement (Fig. $5 B$ ). The average increase (mean \pm SD) in PrV was $29.7 \pm 14.4 \%$, and that for SpI was $28.0 \pm$ $15.3 \%$.

Experiments that have examined the effects of neonatal vibrissa follicle cauterization upon the representation of the vibrissa pad in thalamus and cortex (Durham and Woolsey, 1984) have shown that the central representations of undamaged follicles adjacent to, but not those more distant from, damaged follicles increase in size. In many of the cases we analyzed, the damage to the vibrissa pad was sufficiently extensive so that the "identity" of the remaining follicles was not certain. However, in other cascs (c.g., Fig. 2), the identity of the follicles ablated was readily apparent. We used these cases to evaluate the effect of distance between remaining follicles and the increase in crosssectional areas of their central representations in SpI after damage to the vibrissa pad in utero.

We analyzed 17 cases from ages E- 15 to E-18 in which damage was limited to one or two rows. We collapsed the data across these fetal ages and then related the average percentage increase in patch cross-sectional area with the "distance" between the row analyzed and that removed (Fig. $5 C$ ). As is evident from the figure, there was a significant negative relationship between distance and the percentage increase observed $(F=3.6 ; \mathrm{df}=$ 2,$35 ; p<0.05$ ). Because of this result, we reanalyzed the results from the animals lesioned on E-19, E-20, and P-0 with follicle ablations that were confined to one or two rows. The data from these rats were pooled in the manner described immediately above. In these animals, even the CO-stained patches adjacent to the damaged row or rows did not show significant increases in average cross-sectional area (Fig. 5C).

\section{Relationship between $\mathrm{CO}$-stained patches and primary afferent axons}

Bates and Killackey (1985) have demonstrated clearly that the patches of high $\mathrm{CO}$ reactivity in the trigeminal brainstem complex of both normal perinatal rats and animals that sustained neonatal damage to vibrissa follicles have an exact correspondence with the distribution of primary afferent axon terminals demonstrated by anterograde transport of HRP. We used transganglionic HRP transport to show that this was also the case in rats that sustained follicle cautery in utero. Figure 7 shows adjacent sections through both PrV and SpI processed for HRP reaction product (Fig. $7 A, C$ ) and $\mathrm{CO}$ reactivity (Fig. $7 B, D$ ). Note the cxact correspondence between the patches of primary afferent labeling and $\mathrm{CO}$ reactivity.

\section{Response properties of vibrissa-sensitive $V$ ganglion cells in adult rats that sustained fetal cautery of vibrissa follicles}

We recorded a total of 49 vibrissa-sensitive trigeminal ganglion cells from 12 rats that sustained cautery of vibrissa follicles on

\section{$\leftarrow$}

the results (mean \pm SEM) of the "neighbor" analysis (see Results) for animals lesioned on E-15-18 (solid bars) and animals that sustained vibrissa pad damage on E-19, E-20, or P-0. There was a significant negative relationship between distance and the percentage increase observed for the former group $(F=3.6$; $\mathrm{df}=2,35 ; p<0.05)$, but no significant increases were observed for the latter animals. 

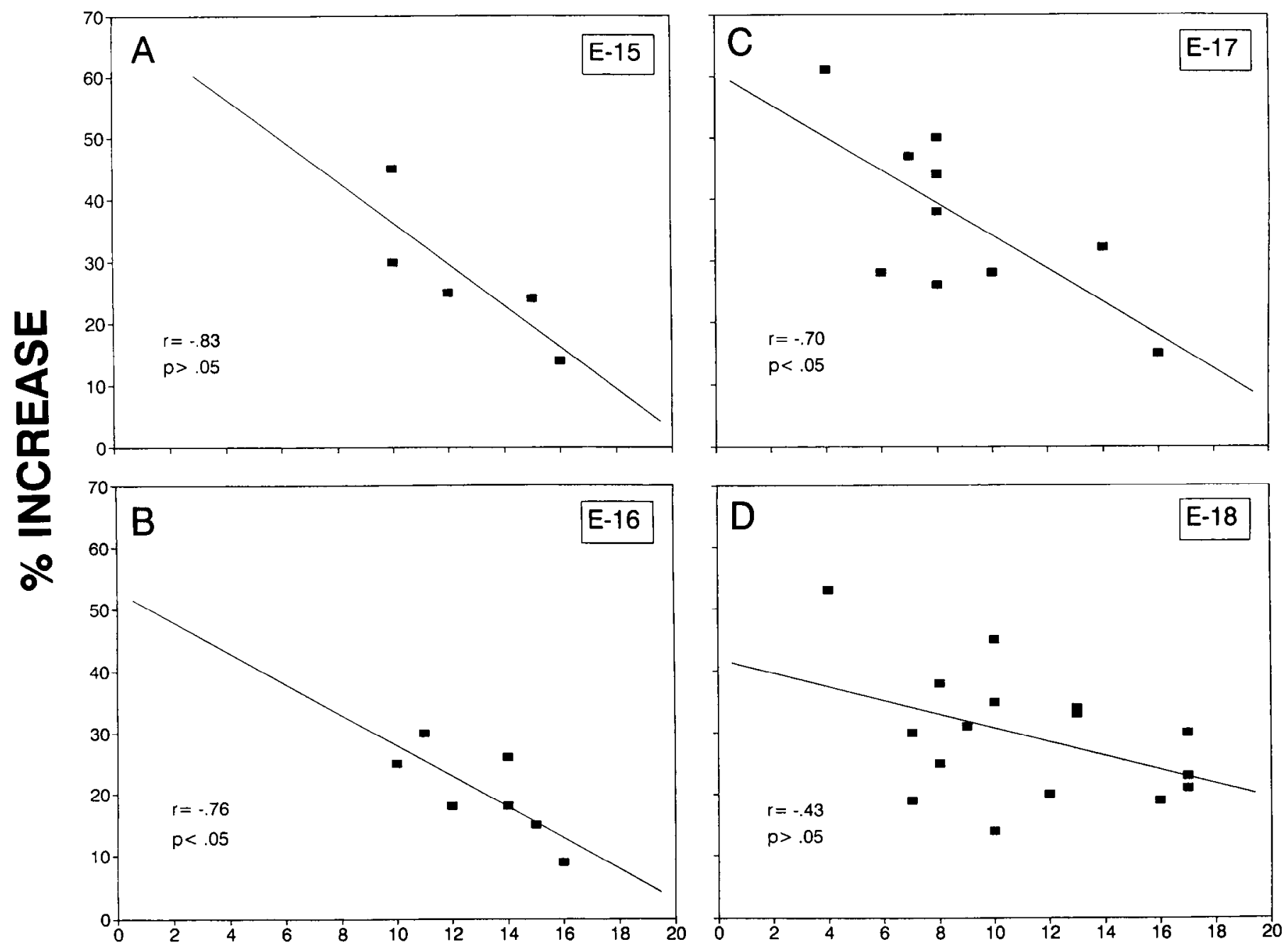

NUMBER OF PATCHES

Figure 6. Scatter plots showing the relationships between number of patches remaining in SpI ipsilateral to the damaged vibrissa pad and the mean increase in average patch size for rats lesioned on E-15-18. The correlation for the animals that sustained lesions on E-15 $(A)$ was -0.82 but was not statistically significant due to the relatively small sample size. The correlation for the rats lesioned on $\mathrm{E}-16(B)$ was $-0.76(p<0.05)$, that for the rats that sustained lesions on E-17 $(C)$ was $-0.70(p<0.05)$, and that for the rats lesioned on E-18 $(D)$ was -0.43 (not significant).

E-17 and survived to adulthood ( $>45 \mathrm{~d}$ of age). All of these neurons were sensitive to deflection of only one mystacial vibrissa (e.g., Fig. $8 A-C$ ). Furthermore, none were activated by stimulation of either the guard hairs between vibrissa follicles or the skin of the vibrissa pad. All of the animals used in these studies had fetal lesions that were verified in two ways. First, all had clear abnormalities in the number and distribution of follicles on the vibrissa pad (e.g., Fig. $8 D$ ) and all had altered cortical $\mathrm{CO}$ patterns corresponding to the vibrissae (Figs. $8 E$, 9). These data are thus consistent with the proposal that the increased size of the representations in PrV and SpI of the vibrissa follicles that remained after fetal lesions was not dependent upon increases in the peripheral receptive fields of vibrissasensitive V ganglion cells.

\section{Effects of fetal vibrissa pad damage upon numbers of fbers in follicle nerves}

We counted the fibers in 11 deep vibrissal nerves from follicles that corresponded to enlarged $\mathrm{CO}$ cortical patches, and their
Table 1. Numbers of animals, average increases in $\mathrm{CO}$ patch size in SpI, and average number of patches remaining on the side ipsilateral to the lesion

\begin{tabular}{lrlr} 
Age & $N$ & $\begin{array}{l}\text { Average increase } \\
\text { in patch size }\end{array}$ & $\begin{array}{l}\text { Number of } \\
\text { patches } \\
\text { remaining }\end{array}$ \\
\hline E-15 & 5 & $27.6 \pm 11.3$ & $12.6 \pm 2.8$ \\
E-16 & 7 & $20.1 \pm 7.2$ & $13.1 \pm 2.2$ \\
E-17 & 10 & $36.9 \pm 13.7$ & $8.9 \pm 3.6$ \\
E-18 & 16 & $29.4 \pm 10.4$ & $11.2 \pm 4.1$ \\
E-19 & 5 & $8.6 \pm 16.1$ & $9.4 \pm 3.4$ \\
E-20 & 10 & $8.9 \pm 16.5$ & $12.4 \pm 2.7$ \\
P-0 & 13 & $6.2 \pm 7.6$ & $11.2 \pm 3.4$
\end{tabular}

Note that maximum possible number of patches (i.e., those measured on the normal side) is 20. Error terms indicate SD. 

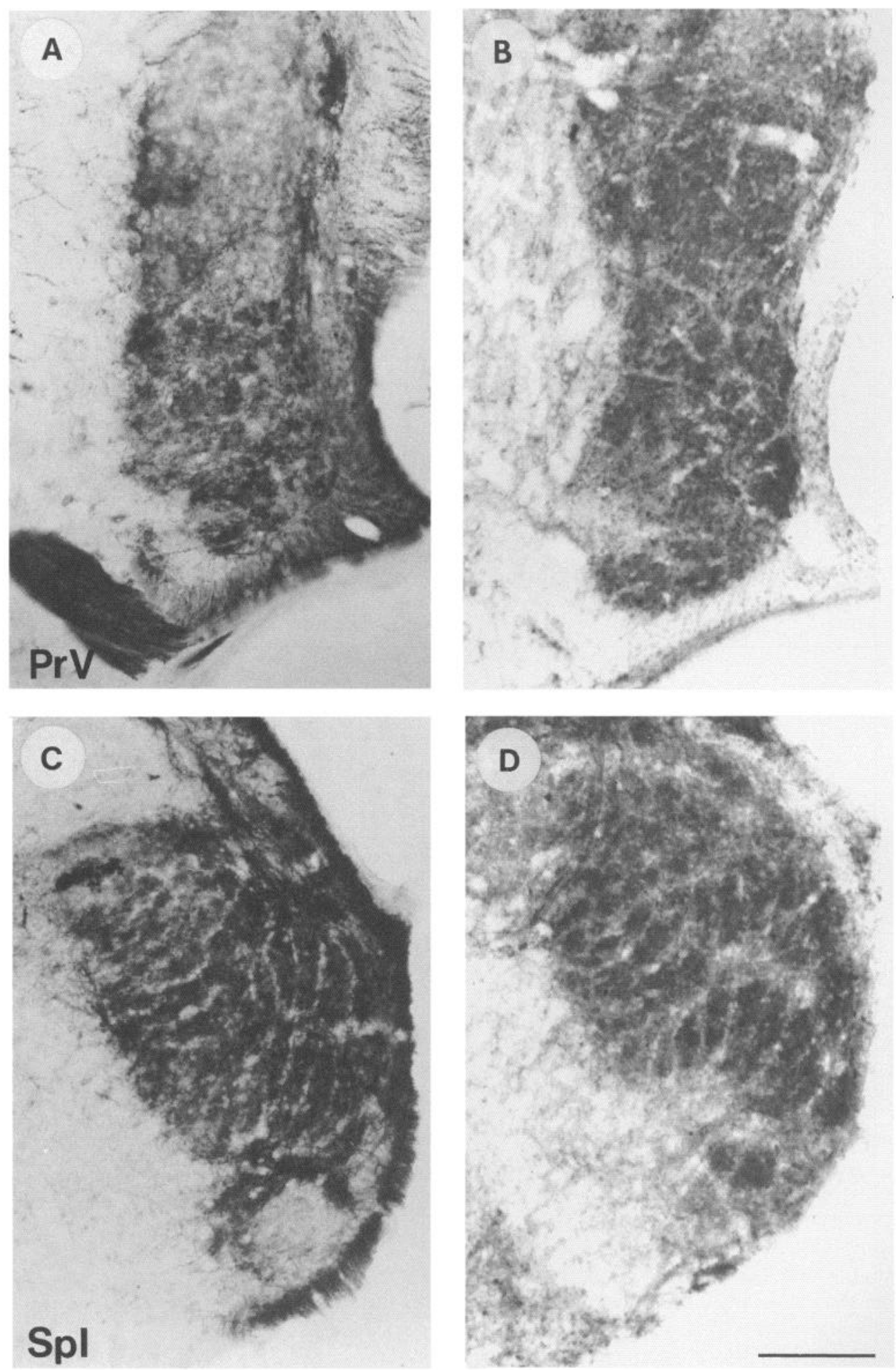

Figure 7. Results from two different cases in which HRP transport was combined with $\mathrm{CO}$ staining. $A$ and $B$ show $\mathrm{HRP}$ reaction product and $\mathrm{CO}$ staining, respectively, in PrV of one rat. Note the correspondence between the two patterns. $C$ and $D$ show data from $\mathrm{SpI}$ from another rat. Again, note the correspondence between the patches of HRP labeling $(C)$ and $C O$ reactivity $(D)$. Scale bar, $200 \mu \mathrm{m}$ for all panels. contralateral normal counterparts. Examples of material used to make the counts are provided in Figure 10, and all counts and the sizes of the corresponding patches of $\mathrm{CO}$ reactivity are shown in Figure 11. The average number of myelinated fibers in the nerves of a follicle with an enlarged patch $(196.8 \pm 27.9)$ was not significantly different from that of its corresponding contralateral normal follicle $(194.6 \pm 25.7)(p>0.05)$. The average size of the patches of $\mathrm{CO}$ reactivity in the cortex contralateral to the lesions in these animals was $0.25 \pm 0.07 \mathrm{~mm}^{2}$. The average for the cortical $\mathrm{CO}$ patches corresponding to the control nerves was $0.16 \pm 0.02 \mathrm{~mm}^{2}(t=4.51 ; \mathrm{df}=10 ; p<$ 0.005 ; paired $t$ test). 
A
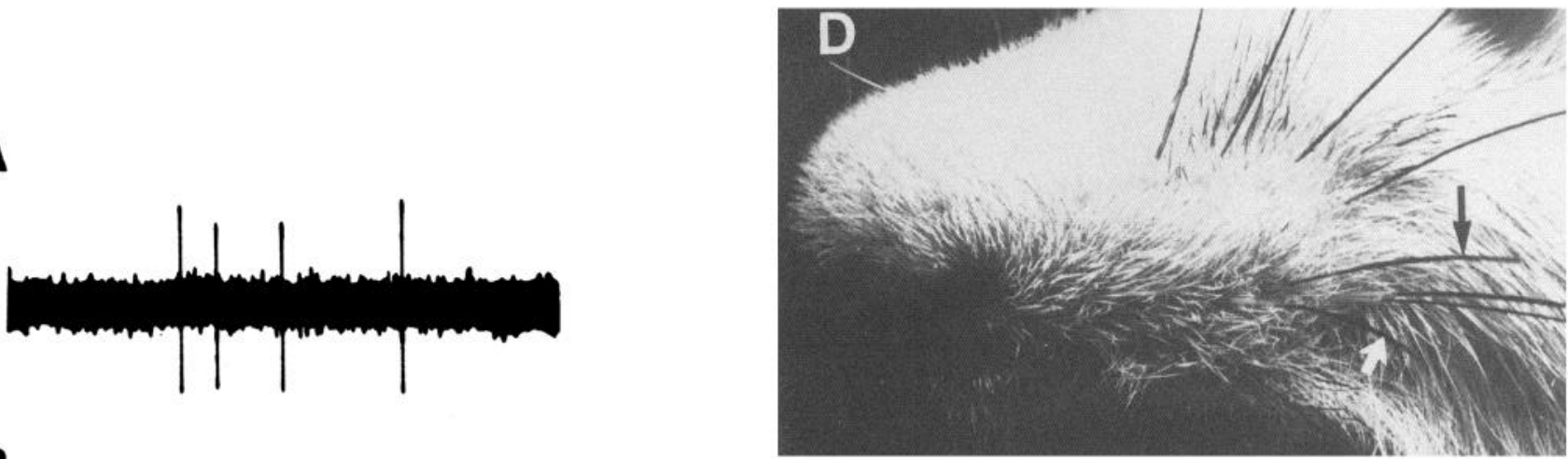

B
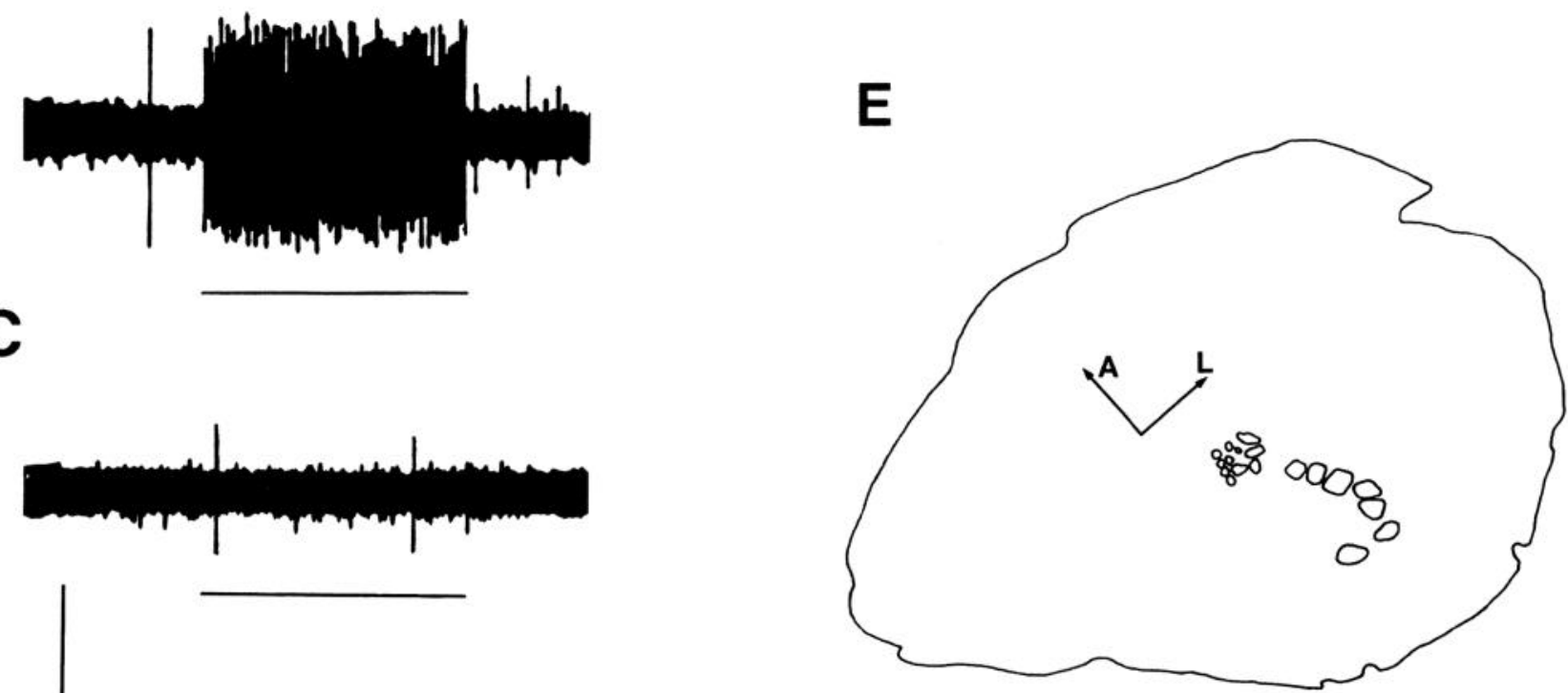

Figure 8. A shows oscillographs from a trigeminal ganglion cell recorded from an adult rat that sustained vibrissa pad damage on E-17. The cell had a low $(<1 \mathrm{~Hz})$ spontaneous firing rate documented by the trace shown in $A$, and it responded $(B)$ only to stimulation of the vibrissa indicated by the solid arrow in the photograph of the vibrissa pad $(D)$. Stimulation of an adjacent vibrissa $(C$, open arrow in $D)$ excited another ganglion cell. Only seven large follicles remained on the damaged vibrissa pad of this animal $(D)$, and reconstruction of the pattern of CO staining in lamina IV of the contralateral cortex reflected this same pattern $(E)$. The calibration for $A-C$ is $1 \mathrm{mV}$ and $1 \mathrm{sec}$. The lines under the traces in $B$ and $C$ indicate the duration of the stimulation. Scale bar in $E, 2 \mathrm{~mm}$; the arrows point toward anterior $(A)$ and lateral $(L)$.

\section{Discussion}

The results described in this article support the conclusion that competitive interactions among vibrissa-related $\mathrm{V}$ primary afferent axons during prenatal development play a substantial role in shaping the central terminal fields of these axons. Using essentially the same assay employed by Belford and Killackey (1980) and Durham and Woolsey (1984), the present study demonstrated that cauterization of vibrissa follicles prior to E-19 resulted in significant increases in the $\mathrm{CO}$ patches corresponding to the remaining follicles.

\section{Lesion-induced primary afferent reorganization}

There is considerable evidence that undamaged primary afferent axons in perinatal rodents can expand their terminal fields after peripheral nerve lesions and, further, that there is a limited sensitive or critical period after which such lesions no longer produce reorganization of the central axons of undamaged sensory ganglion cells.
Rhoades et al. (1989) transected the infraorbital nerve (ION, the $\mathrm{V}$ branch innervating the vibrissa follicles) in rats on E-16 and used transganglionic tracing with HRP when these animals reached adulthood to demonstrate expanded central territories for undamaged mandibular axons. Similar lesions in newborn rats produced no alterations in the central terminal distributions of undamaged mandibular $\mathrm{V}$ branches (Jacquin and Rhoades, 1985). Rhoades et al. (1983) showed that transection of the ION in newborn hamsters did result in expansion of the terminal fields of undamaged mandibular primary afferents. The hamster is born after a $16 \mathrm{~d}$ gestation period (that for the rat is $21 \mathrm{~d}$ ), and the $\mathrm{V}$ system may be less mature in newborn animals of this species. Accordingly, Jacquin and Rhoades (1987) transected the ION in 5-d-old hamsters and found that mandibular sensory afferents remained restricted to their normal territories. Fitzgerald (1985) and Fitzgerald and Vrbová (1985) have reported expansion of the central terminal field of the saphenous nerve after neonatal sciatic nerve damage in rat. The fact that postnatal peripheral nerve lesions in the rat result in primary 

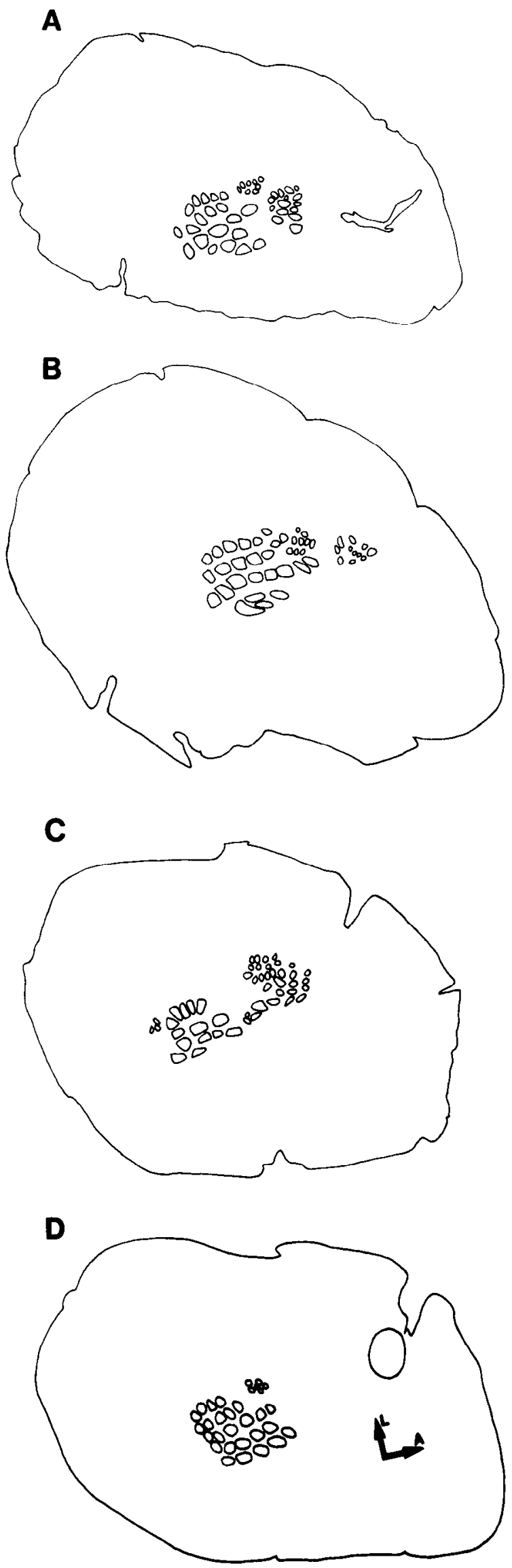

afferent reorganization in the spinal cord, but not the $\mathrm{V}$ brainstem complex, may reflect the generally rostrocaudal gradient of CNS development (see Ayer-LeLievre and Seiger, 1984, for additional discussion).

Experiments in nonmammalian vertebrates have also demonstrated that peripheral damage during early development can alter the central terminal fields of undamaged axons. In general, the manipulation used in these experiments has been ablation of selected dorsal root ganglia rather than transection of peripheral nerves. Frank and his coworkers (Frank and Westerfield, 1982; Smith and Frank, 1988; Mendelson and Frank, 1989; Frank and Mendelson, 1990) have shown that deletions of dorsal root ganglia result in altered central terminal fields by undamaged ganglion cells.

\section{Do the larger patches of $C O$ reactivity represent enlarged} central terminal fields by vibrissa-related primary afferents?

The present results and the previous findings of Bates and Killackey (1985) provide strong support for the conclusion that metabolic stains such as $\mathrm{CO}$ and $\mathrm{SDH}$ provide a very accurate representation of the clustered terminal arbors of vibrissa-related trigeminal primary afferent terminations. However, since $\mathrm{CO}$ staining is an indirect method of demonstrating the size and location of these terminal arbors, another potential interpretation of the present results must be considered.

The effect observed in the brainstem might reflect a reduction in the overlap of the central arbors of $\mathrm{V}$ axons innervating a given follicle. Indeed, Jensen and Killackey (1987) suggested that one of the consequences of neonatal ION damage upon individual thalamocortical axons innervating the rat's somatosensory postcromedial barrel subfield was a reduction in the overlap of fibers innervating a given small portion of the cortex. The results of Shatz and her coworkers (Stretavan and Shatz. 1986; Garraghty et al., 1988; Roe et al., 1989) suggest an analogous result in the LGNd after removal of one eye in fetal cats. There is an expansion of the terminal arbors of $\mathrm{Y}$-cell axons, but normally sized $\mathrm{X}$-axons are sometimes located in inappropriate parts of the LGNd.

Two aspects of the present data suggest that a reduction in the overlap of the central arbors of primary afferents innervating a given vibrissa follicle is not a likely explanation of the changes observed in the brainstem. First, if overlap of fibers innervating a given vibrissa was reduced, it would likely follow that the segmented patterns of both CO reactivity and HRP-labeled primary afferent terminals would be degraded (i.e., blurred). This is, in fact, what occurs in cortex after neonatal ION transection (Jensen and Killackcy, 1987).

The patterns observed with both $\mathrm{CO}$ staining and HRP labeling appeared as well defined in the experimental animals as in normal rats. We thus believe that $\mathrm{CO}$ staining in animals with fetal vibrissa follicle damage, as in normal animals, provides an accurate representation of the clustering of primary afferent axons in the trigeminal brainstem complex.

$\leftarrow$

Figure 9. Reconstructions of vibrissa-related CO patterns in a control cortex $(D)$ and in cortices contralateral to the damaged vibrissa pads of three rats that were used in the electrophysiological experiments $(A-C)$. The arrows in the reconstruction in $D$ point toward anterior $(A)$ and lateral $(L)$, and they also apply to all other panels. Scale bar, $2 \mathrm{~mm}$ for all panels. 

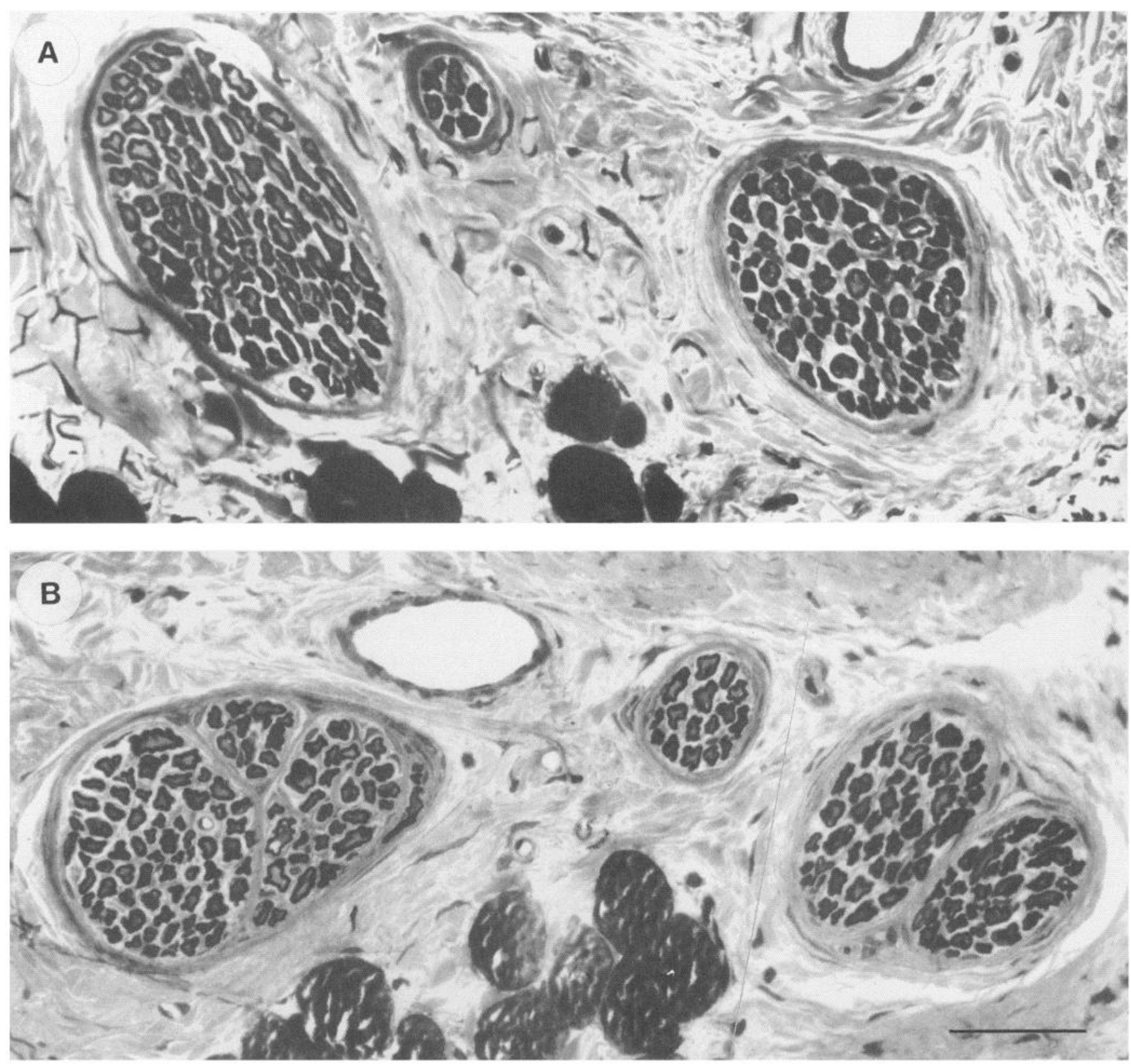

Figure 10. Photomicrographs of the follicle nerves innervating the $\mathrm{C} 2$ vibrissa follicle on the intact $(A)$ and damaged $(B)$ sides of the face. The nerve on the intact side contained 199 myelinated axons, and that on the damaged side contained 206 myelinated fibers. Scale bar, $50 \mu \mathrm{m}$ for both panels.

Does the expansion of the central terminations of undamaged primary afferents necessarily reflect interaxonal competition?

Competitive interactions among the axons innervating a given target are usually imputed when removal (Scott, 1984; Mendelson and Frank, 1989) or reduction in the activity (Guillery, 1972a; Sherman and Spear, 1982) of one subset of afferents results in an increase in the terminal arborizations of fibers that remain or retain normal patterns of activity. The underlying assumption in the interpretation of such results as reflecting competition is that subpopulations of afferent axons compete for some limited commodity at the target (e.g., trophic substance, synaptic sites). We believe that the present results reflect such competitive interactions among the central axons of vi- brissa-related trigeminal primary afferents, but there are other possible interpretations of our findings.

Smith and Frank (1988) have interpreted the central primary afferent reorganization that follows neural crest lesions in bullfrog to reflect a process that they have referred to as "respecification." They have suggested that the central primary afferent reorganization that follows dorsal root ganglion ablation in bullfrog tadpoles may be a secondary result of peripheral axonal sprouting by these same ganglion cells. Changes in the location of the central arbors of these axons may simply reflect their new peripheral targets. Most recently, Mendelson and Frank (1989) demonstrated that removal of the brachial dorsal root ganglion in developing frogs resulted in a small number of neurons in the adjacent dorsal root ganglion "sprouting" into the dener- 


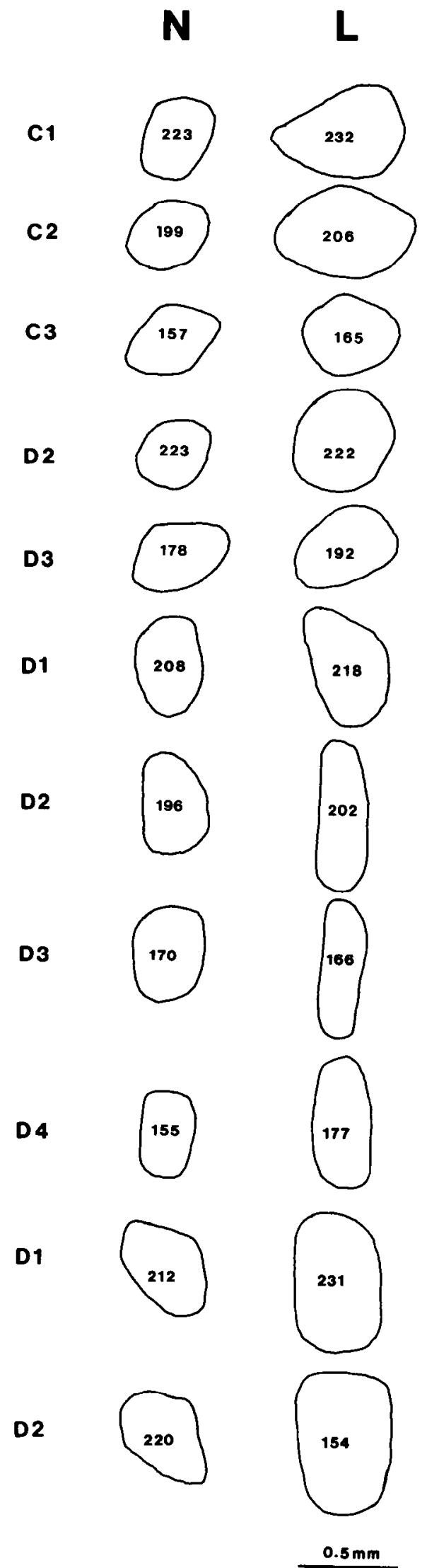

Figure 11. Counts of myelinated nerve fibers from 11 follicle nerves from damaged vibrissa pads (right column) and matched nerves from the intact vibrissa pads. The nerves counted are designated in the left column. The closed contours surrounding each nerve count are drawings of the CO-stained patches corresponding to the follicle in question in vated triceps muscle and corresponding part of the spinal cord. Importantly, their results indicated that these primary afferents did not develop abnormally large central arbors, but rather central terminations that were appropriate with respect to their new peripheral target. Thus, while competition among primary afferent axons may play a role in the development of primary afferent projections to the periphery (Scott, 1984; Mendelson and Frank, 1989), it need not be imputed in either their normal central development or their lesion-induced reorganization.

It is unlikely that the central changes observed in the present experiment reflect peripheral respecification of the type described above. All of the vibrissa-sensitive ganglion cells recorded in the fetally lesioned rats responded to deflection of only one whisker. This is also the case in normal adult animals (Zucker and Welker, 1969; Jacquin et al., 1986). It is also worth noting the sizes of the peripheral receptive fields of $\mathrm{V}$ ganglion cells appear mature at, or prior to, the time at which most of the lesions in this study were carried out. Chiaia et al. (1990) recorded from $\mathrm{V}$ ganglion cells in rats as young as E-16. Nearly all of the primary afferents that could be excited by stimulation of a vibrissa follicle at this age had receptive fields restricted to that follicle.

The observation that fetal cauterization of vibrissa follicles did not lead to the development of abnormal receptive fields by the vibrissa-sensitive axons that remained may seem somewhat surprising in view of the fact that both fetal (Chiaia et al., 1989) and neonatal transection of the ION (Jacquin et al., 1986; Rhoades et al., 1987; Chiaia et al., 1988) does result in the development of abnormal peripheral projections by undamaged neurons. However, a major difference between those studies and the present report is that damage in this study was restricted to the periphery rather than to the entire ION. The abnormal receptive fields that developed in those studies most likely occurred as a result of misrouting of regenerate axons and invasion of denervated peripheral territory by undamaged axons from other $\mathrm{V}$ branches (Rhoades et al., 1987). The fact that the periphery was damaged directly in the present study probably reduced the likelihood of peripheral sprouting by undamaged axons. Furthermore, regenerate axons may have been inhibited from innervating intact vibrissa follicles because those that remained were already supplied by their normal complement of $\mathrm{V}$ axons. The suggestion is supported by the result that undamaged follicles on the damaged vibrissa pad were not innervated by an abnormal number of deep vibrissal nerve fibers.

Does the critical period for central reorganization of vibrissa-related primary afferents depend upon overlap of these fibers at the time of our lesions?

The present results suggest a strong temporal correspondence between the development of segregation of the central terminal

the contralateral cortex. The average number (mean \pm SD) of myelinated fibers from the follicles in the damaged vibrissa pads was $196.8 \pm$ 27.9 , and that for the corresponding contralateral nerves $194.6 \pm 25.7$ $(p>0.05)$. The average size of the cortical patches of CO reactivity corresponding to the nerves counted in the lesioned vibrissa pads was $0.25 \pm 0.07 \mathrm{~mm}^{2}$. The average for the cortical $\mathrm{CO}$ patches corresponding to the control nerves was $0.16 \pm 0.02 \mathrm{~mm}^{2}(t=4.51 ; \mathrm{df}=10, p<$ 0.005 , paired $t$ test). 
arbors of primary afferents innervating different vibrissa follicles and time during which damage to some primary afferents results in increased terminal fields for those that remain. While a vibrissa-related pattern was not fully developed until E-20, some segmentation could be seen in animals killed on E-19. Lesions made later than E-18 failed to produce a significant increase in the size of the CO patches that remained in these animals. This result raises the possibility that some overlap of axon arbors may provide the necessary substrate for interaxonal competition in this system.

It is important to note that overlap between populations of afferent axons is not a necessary condition for competition of these populations. This was demonstrated quite clearly by LeVay et al. (1980), who showed that monocular deprivation of a monkey beginning at $5 \frac{1}{2} 2$ weeks of age resulted in a substantial expansion of the ocular dominance columns related to the nondeprived eye. At $5 \frac{1 / 2}{2}$ weeks of age, ocular dominance columns in monkey show a nearly adult degree of segregation.

There is also evidence that overlap of terminal fields is not a necessary condition for alterations in the central arbors of undamaged primary afferents after peripheral lesions. Neonatal ION transections in hamsters result in an increase in the terminal zone of undamaged mandibular primary afferents (Rhoades et al., 1983). Transganglionic tracing in newborn hamsters indicated no central overlap between mandibular and ION fibers (Jacquin and Rhoades, 1985). Similarly, expansion of the central terminal field of the axons that supply the auriculotemporal sinus hair occurs after postnatal ION lesions (Waite, 1990). By birth, the $\mathrm{CO}$ patch related to this whisker is clearly segregated from those of the mystacial vibrissae.

All of the results described in the preceding paragraph were obtained in preparations where large portions of the periphery were denervated. Such damage has been shown repeatedly to result in substantial reorganization of the peripheral processes of primary afferent neurons (e.g., Frank and Westerfield, 1982; Jackson and Diamond, 1984; Scott, 1984; Kinnman and Aldskogius, 1986; Rhoades et al., 1987) that may in turn lead to respecification of the central arbors of these cells (Smith and Frank, 1988). It thus seems likely that the results of these experiments may not be directly comparable to those of the present study.

One other caveat should be raised with respect to the relationship between the development of vibrissa-related segregation in the brainstem as reflected by $\mathrm{CO}$ staining and the sensitive period for lesion-induced increases in the central arbors of undamaged primary afferents. There may be a substantial delay between the time when vibrissa-related primary afferents develop a segmented pattern and that when a corresponding CO pattern emerges in the brainstem. This is certainly the case with respect to the development of thalamocortical afferents and SDH patterns in the rodent somatosensory cortex (compare the results of Erzurumlu and Jhaveri, 1990, with those of Killackey and Belford, 1979), and preliminary results (Crissman et al., 1989; R. W. Rhoades, unpublished observations) indicate that this is also true in the brainstem.

In summary, the present results indicate a temporal correlation between the normal development of vibrissa-related segmentation in the brainstem and the ability of peripheral lesions to alter the size of the terminal arbors of undamaged afferents. This correlation and its potential implications must be viewed with caution until normal trigeminal primary afferent development is described with direct axonal tracing techniques.

\section{Summary}

The present results support the conclusion that interaxonal competition in the brainstem plays an important role in shaping the terminal arbors of these fibers. There is a period during prenatal development when damage to primary afferents leads to reorganization (probably increases in size) of undamaged axons that is independent of any change in the peripheral innervation patterns of these fibers. Finally, the period when such effects can be induced has at least a rough correspondence with that during which the central arbors of axons innervating different vibrissa follicles may overlap in the brainstem.

\section{References}

Ayer-LeLievre CS, Seiger A (1984) Development of substance Pimmunoreactive neurons in cranial sensory ganglia of the rat. Int $\mathbf{J}$ Dev Neurosci 2:451-463.

Bates CA, Killackey HP (1982) The organization of the neonatal rat's brainstem trigeminal complex and its role in the formation of central trigeminal patterns. J Comp Neurol 240:265-287.

Belford GR, Killackey HP (1989) The sensitive period in the development of the trigeminal system of the neonatal rat. J Comp Neurol 193:335-350.

Bennett M, Ho S, Lavidis N (1986) Competition between segmental nerves at end-plates in rat gastrocnemius muscle during loss of polyneuronal innervation. J Physiol (Lond) 381:351-376.

Betz JH, Caldwell JH, Ribchester RR (1980) The effects of partial denervation at birth on the development of muscle fibers and motor units in rat lumbrical muscle. J Physiol (Lond) 303:265-279.

Chalupa LM, Williams RW (1984) Organization of the cat's lateral geniculate nucleus following interruption of prenatal binocular competition. Hum Neurobiol 3:103-107.

Chiaia NL, Allen Z, Carlson E, Macdonald G, Rhoades RW (1988) Neonatal infraorbital nerve transection in rat results in peripheral trigeminal sprouting. J Comp Neurol 274:101-114.

Chiaia NL, Macdonald GJ, Bauer WR, Rhoades RW (1989) Effects of fetal infraorbital nerve damage upon the projections of individual trigeminal primary afferent neurons. Soc Neurosci Abstr 15:92.

Chiaia NL, Macdonald GJ, Rhoades RW (1990) Receptive field properties of trigeminal ganglion cells in fetal rats. Soc Neurosci Abstr 16: 830.

Coleman L-A, Beazley LD (1989) Expanded retinofugal projections to the dorsal lateral geniculate nucleus and superior colliculus after unilateral enucleation in the wallaby Setonix brachyurus, quokka. Dev Brain Res 48:273-291.

Crissman RS, Parsons LL, Chiaia NL, Rhoades RW (1989) The primary afferent innervation of the trigeminal brainstem complex of fetal rats as demonstrated by anterograde transport of DI-I. Soc Neurosci Abstr 15:873.

Durham D, Woolsey TA (1984) Effects of neonatal whisker lesions on mouse central trigeminal pathways. J Comp Neurol 223:424-447.

Erzurumlu RS, Jhaveri S (1990) Thalamic afferent segregation precedes barrel formation in the rat SI cortex. Soc Neurosci Abstr 16: 1214.

Erzurumlu RS, Killackey HP (1983) Development of order in the rat trigeminal system. J Comp Neurol 213:365-380.

Fitzgerald $M$ (1985) The sprouting of saphenous nerve terminals in the spinal cord following early postnatal sciatic nerve section in the rat. J Comp Neurol 240:407-413.

Fitzgerald M, Vrbová G (1985) Plasticity of acid phosphatage (FRAP) afferent terminal fields and of dorsal horn cell growth in the neonatal rat. J Comp Neurol 240:414-422.

Frank E, Mendelson B (1990) Specification of synaptic connections between sensory and motor neurons in the developing spinal cord. $J$ Neurobiol 21:33-50.

Frank E, Westerfield M (1982) The formation of appropriate central and peripheral connexions by foreign sensory neurones of the bullfrog. J Physiol (Lond) 324:495-505.

Garraghty PE, Shatz CJ, Sretavan DW, Sur M (1988) Axon arbors of $X$ and $Y$ retinal ganglion cells are differentially affected by prenatal disruption of binocular inputs. Proc Natl Acad Sci USA 85:73617365 . 
Guillery RW (1972a) Binocular competition in the control of geniculate cell growth. J Comp Neurol 144:117-130.

Guillery RW (1972b) Experiments to determine whether retinogeniculate axons can form translaminar collateral sprouts in the dorsal lateral geniculate nucleus of the cat. J Comp Neurol 146:407-420.

Jackson PC, Diamond J (1984) Temporal and spatial constraints on the collateral sprouting of low-threshold mechanosensory nerves in the skin of rats. J Comp Neurol 226:336-345.

Jacquin MF, Rhoades RW (1985) Effects of nconatal infraorbital lesions upon central trigeminal primary afferent projections in rat and hamster. J Comp Neurol 235:129-143.

Jacquin MF, Rhoades RW (1987) Development and plasticity in hamster trigeminal primary afferent projections. Dev Brain Res 31:161175.

Jacquin MF, Renehan WE, Klein BG, Mooney RD, Rhoades RW (1986) Functional consequences of neonatal infraorbital nerve section in rat trigeminal ganglion. J Neurosci 6:3706-3720.

Jensen KF, Killackey HP (1987) Terminal arbors of axons projecting to the somatosensory cortex of the adult rat. II. The altered morphology of thalamocortical afferents following neonatal infraorbital nerve cut. J Neurosci 7:3544-3553.

Killackey HP, Belford GR (1979) The formation of afferent patterns in the somatosensory cortex of the nconatal rat. J Comp Neurol 183: 285-304.

Kinnman E, Aldskogius H (1986) Collateral sprouting of sensory axons in the glabrous skin of the hindpaw after chronic sciatic nerve lesion in adult and neonatal rats: a morphological study. Brain Res 377:7382.

LeVay S, Wiesel TN, Hubel DH (1980) The development of ocular dominance columns in normal and visually deprived monkeys. J Comp Neurol 191:1-51.

Lund RD, Lund JS (1971) Synaptic adjustment after deafferent action of the superior colliculus of the rat. Science 171:804-807.

Mendelson B, Frank E (1989) Role of competition among sensory neurons in regulation of pattern of innervation at their central and peripheral targets. J Neurophysiol 62:1189-1200.

Mesulam M-M (1978) Tetramethylbenzidine for horseradish pcroxidase neurohistochemistry: a non-carcinogenic blue reaction product with superior sensitivity for visualizing neural afferents and efferents. J Histochem Cytochem 26:106-117.

Rakic P (1981) Development of visual centers in the primate brain depends on binocular competition before birth. Science 214:928-931.
Rhoades RW, Fiore JM, Math MF, Jacquin MF (1983) Reorganization of trigeminal primary afferents following neonatal infraorbital nerve section in hamster. Dev Brain Res 7:337-342.

Rhoades RW, Chiaia NL, Mooney RD, Klein BG, Renehan WE, Jacquin MF (1987) Reorganization of the peripheral projections of the trigeminal ganglion following neonatal transection of the infraorbital nerve. Somatosens Res 5:35-62.

Rhoades RW, Chiaia NL, Macdonald GJ, Jacquin MF (1989) Effect of fetal infraorbital nerve transection upon trigeminal primary afferent projections in the rat. J Comp Neurol 287:82-97.

Roe AW, Garraghty PE, Shatz CJ, Sretavan DW, Sur M (1989) Developmental interactions that regulate the size and location of retinogeniculate X and Y axon arbors. Invest Ophthalmol Vis Sci 30:296.

Scott SA (1984) The effects of neural crest deletions on the development of sensory innervation patterns in embryonic chick hind limb. J Physiol (Lond) 352:285-304

Sherman SM, Spear PD (1982) Organization of visual pathways in normal and visually deprived cats. Physiol Rev 62:738-855.

Smith CL, Frank E (1988) Peripheral specification of sensory connections in the spinal cord. Brain Behav Evol 31:227-242.

Sretavan DW, Shatz CJ (1986) Prenatal development of cat retinogeniculate axon arbors in the absence of binocular interactions. J Neurosci 6:990-1003.

Ungewitter LH (1951) A urea silver nitrate method for nerve fibers and nerve endings. Stain Technol 26:73-76.

Waite PME (1990) Plasticity in cranial somatosensory pathways. Processing in mammalian auditory and tactile systems, pp 237-251. New York: Liss.

Wiesel TN, Hubel DH (1963) Effects of visual deprivation on morphology and physiology of cells in the cat's lateral geniculate body. $\mathrm{J}$ Neurophysiol 26:978-993.

Wong-Riley M (1979) Changes in the visual system of monocularly sutured or enucleated cats demonstrable with cytochrome oxidase histochemistry. Brain Res 160:134-138.

Woolsey TA, Wann JR (1976) Areal changes in mouse cortical barrels following vibrissal damage at different postnatal ages. J Comp Neurol 170:53-66.

Zucker E, Welker WI (1969) Coding of somatic sensory input by vibrissae neurons in the rat's trigeminal ganglion. Brain Res 12:138156. 